\title{
Bias of Least Squares Approaches for Diffusion Tensor Estimation from Array Coils in DT-MRI
}

\author{
Antonio Tristán-Vega ${ }^{1}$, Carl-Fredrik Westin ${ }^{2}$, and Santiago Aja-Fernández ${ }^{1}$ \\ 1 Laboratory of Image Processing. Universidad de Valladolid, Spain \\ 2 LMI. Brigham and Women's Hospital, Boston \\ atriveg@lpi.tel.uva.es, westin@bwh.harvard.edu, sanaja@tel.uva.es
}

\begin{abstract}
Least Squares (LS) and its weighted version are standard techniques to estimate the Diffusion Tensor (DT) from Diffusion Weighted Images (DWI). They require to linearize the problem by computing the logarithm of the DWI. For the single-coil Rician noise model it has been shown that this model does not introduce a significant bias, but for multiple array coils and parallel imaging, the noise cannot longer be modeled as Rician. As a result the validity of LS approaches is not assured. An analytical study of noise statistics for a multiple coil system is carried out, together with the Weighted LS formulation and noise analysis for this model. Results show that the bias in the computation of the components of the DT may be comparable to their variance in many cases, stressing the importance of unbiased filtering previous to DT estimation.
\end{abstract}

\section{Introduction}

Least Squares (LS) techniques have become the standard to estimate the Diffusion Tensor (DT) in DT-MRI [1. Although other approaches are possible 2], LS are fast and robust, and they show optimal properties (minimum variance) when Weighted Least Squares (WLS) is used [1]. For single-coil acquisition systems where the noise is Rician distributed [3], the estimation is nearly unbiased, so WLS is in this case the Best Linear Unbiased Estimator (BLUE). With the advent of multiple receiver coils for similtaneous acquisition and parallel reconstruction schemes (pMRI), the noise in the Diffusion Weighted Images (DWI) is no longer Rician 456] and the properties of WLS have not been analyzed for these composite signals. In addition, although the importance of the bias in DWI has been studied in filtering problems, 78910, the necessity of bias removal for DT estimation has not been either thoroughly analyzed to the very best of our knowledge. Therefore the main contributions of the present paper are: first, we theoretically justify the conclusions of [1] regarding the bias and variance of $\log -$ Rician distributed signals; for array coils, the $\log -$ non-central $\chi$ model has been suggested [5], so we derive both analytical expressions and practical approximations for its statistical characterization. Based on this analysis, we secondly extend the work in [1] to multiple coils DT-MRI, showing that it admits the same WLS formulation. Third we show that, as opposed to the case of Rician noise, the bias in WLS estimation is relevant with array coils as 
a consequence of the estimation of the composite magnitude signal by means of Sum of Squares (SoS). This implies that the analysis may be generalized to some pMRI reconstruction schemes based on SoS, such as the popular GRAPPA technique. At the same time, the need for unbiased filtering before DT estimation is evidenced.

\section{Statistics of Noise in the Log-Domain}

Under the assumption of narrow pulses, and for mono-compartment Gaussian diffusion, the tensor model relates the DWI signal $A_{i}$ for each gradient direction $i$ to the T2 baseline image $A_{0}$ following the Stejskal-Tanner equation [11]:

$$
A_{i}=A_{0} \exp \left(-b g_{i}^{T} D g_{i}\right) \Leftrightarrow \log \left(A_{0}\right)-\log \left(A_{i}\right)=b g_{i}^{T} D g_{i}, 1 \leq i \leq N
$$

where $g_{i}$ are the $N$ gradient directions, $b$ is the diffusion weighting parameter, and $D$ is the DT. Assuming $A_{i}$ real, for a single-coil acquisition the received signal $M_{i}$ is the Rician distributed envelope of a complex Gaussian process [3]:

$$
M_{i}=\sqrt{\left(A_{i}+n_{c, i}^{2}\right)^{2}+n_{s, i}^{2}}
$$

where $n_{c, i}$ and $n_{s, i}$ are independent Gaussian processes with zero mean and standard deviation $\sigma$. In phased array systems with multiple coils -assuming no subsampling in the $\mathbf{k}$-space--, the composite magnitude signal after sum-ofsquares combination may be modeled as [45]:

$$
M_{i}=\sqrt{\sum_{l=1}^{L}\left(A_{i, l}+n_{c, i, l}^{2}\right)^{2}+n_{s, i, l}^{2}}
$$

for $L$ receiving coils. This correponds to a non-central $\chi$ distribution. Note that the Rician distribution is a particular case for $L=1$, so the latter may be seen as a general model for single and multiple coils acquisition schemes. However, as shown in Section 3, the statistics of interest are those related to the logarithm of $M$; the following expressions for the mean and variance may be proved 1 :

$$
\begin{aligned}
E\{\log (M)\}= & \frac{1}{2} \log \left(2 \sigma_{n}^{2}\right)+\frac{1}{2} \frac{a}{L}{ }_{2} F_{2}(1,1: 2,1+L ;-a)+\frac{1}{2} \psi(L) ; \\
\operatorname{Var}\{\log (M)\}= & \frac{1}{4}\left[\widetilde{\mathrm{N}}_{L}(a)-2 \log \left(2 \sigma^{2}\right) \frac{a}{L}{ }_{2} F_{2}(1,1: 2,1+L ;-a)\right. \\
& \left.-\left(\psi(L)+\frac{a}{L}{ }_{2} F_{2}(1,1: 2,1+L ;-a)\right)^{2}\right]
\end{aligned}
$$

with:

$$
\widetilde{\mathrm{N}}_{k}(x)=e^{-x} \sum_{p=0}^{\infty} \frac{x^{p}}{\Gamma(p+1)}\left[(\psi(p+k))^{2}+\psi^{1)}(p+k)\right],
$$

${ }^{1}$ We have to omit the detailed proof here due to space constraints. 
where $\psi(x)$ is the polygamma function and $\psi^{1)}(x)$ is its first derivative. For convenience we have dropped the $i$ subindex and used the notation $A_{L}^{2}=\sum_{l=1}^{L} A_{l}^{2}$ and $a=A_{L}^{2} / 2 \sigma^{2}$. Eq. (4) generalizes the expression given in [1, eq. (11)] for the mean of the $\log$-Rician distribution, while eq. (5) has not been previously reported to the best of our knowledge. The complexity of these expressions does not allow a detailed analysis, so we use practical approximations. To do so, let us compute the Taylor series expansion of $\log (M)$ for $\sigma \ll A_{L}$; without loss of generality, we may assume that $A_{L}^{2}=L A^{2}$, and then:

$$
\begin{aligned}
\log (M) & =\log \left(A_{L}\right)+\frac{1}{A L} \sum_{i=1}^{L} n_{c, l}+\frac{1}{2 A^{2} L^{2}} \sum_{k=1}^{L} \sum_{l=1}^{L}\left(L \delta_{k l}-2\right) n_{c, k} n_{c, l} \\
& +\frac{1}{2 L A^{2}} \sum_{l=1}^{L} n_{s, l}^{2}+\mathcal{O}\left(\frac{\sigma^{3}}{A^{3}}\right)
\end{aligned}
$$

with $\delta$ the Kronecker delta function. From eq. (7) it follows:

$$
E\{\log (M)\}=\log \left(A_{L}\right)+(L-1) \frac{\sigma^{2}}{A_{L}^{2}}+\mathcal{O}\left(\frac{\sigma^{3}}{A^{3}}\right)
$$

In fact, since the expectation of all terms in $\mathcal{O}\left(\sigma^{3} / A^{3}\right)$ represent odd-order moments of Gaussians, we could have written $\mathcal{O}\left(\sigma^{4} / A^{4}\right)$ instead. The Mean Squared Error (MSE) in the estimation may be computed as the variance plus the squared bias, so we must compute the expansion of the variance to order 4 (comparable to bias $^{2}=\mathcal{O}\left(\sigma^{4} / A_{L}^{4}\right) \equiv \mathcal{O}\left(a^{-2}\right)$ ), being necessary to compute the expansion of $\log (M)$ to order 3 . After some calculations, it yields:

$$
\begin{gathered}
\operatorname{Var}\{\log (M)\}=\frac{1}{2} a^{-1}-\frac{3 L-4}{4} a^{-2}+\mathcal{O}\left(a^{-3}\right) \\
\operatorname{bias}^{2}\{\log (M)\}=\frac{(L-1)^{2}}{4} a^{-2}+\mathcal{O}\left(a^{-3}\right)
\end{gathered}
$$

For the Rician case $(L=1)$, the squared bias is in the order of $\mathcal{O}\left(a^{-3}\right)=$ $\mathcal{O}\left(\sigma^{6} / A_{L}^{6}\right)$, so it is not so important, as has been noted in [1]. For $L>1$, the bias may be important if $L$ is in the same order of magnitude as $A_{L} / \sigma$.

\section{Tensor Fitting Based on Weighted Least Squares}

From the linearized version of the Stejskal-Tanner model in eq. (1), the estimation of the 6 free coefficients of the DT may be seen as a WLS problem [1]:

$$
\begin{aligned}
\log \left(A_{0}\right)-\log \left(M_{i}\right)= & {\left[g_{i, 1}^{2}, 2 g_{i, 1} g_{i, 2}, 2 g_{i, 1} g_{i, 3}, g_{i, 2}^{2}, 2 g_{i, 2} g_{i, 3}, g_{i, 3}^{2}\right] } \\
& \cdot\left[b D_{11}, b D_{12}, b D_{13}, b D_{22}, b D_{23}, b D_{33}\right]^{T}+\epsilon_{i}
\end{aligned}
$$

As opposed to [1, we do not include $A_{0}$ in the estimation, i.e., we consider that $A_{0}$ is known without any uncertainty $\left(A_{0}=M_{0}\right)$; since the baseline is generally far 
less noisy than the gradient images (its value of $a$ is much higher), and moreover several baseline images are often available, the error due to $A_{0}$ is negligible and therefore it does not affect our analysis. Nevertheless, a similar analysis to that here presented may be carried out including $A_{0}$ in the estimation with almost identical conclusions. The WLS problem may then be written:

$$
G^{T} W(\mathbf{Y}-G \mathbf{X})=0 \Rightarrow \mathbf{X}=\left(G^{T} W G\right)^{-1} G^{T} W \mathbf{Y}
$$

where $\mathbf{Y}$ is an $N \times 1$ vector representing each of the DWI data $\log \left(M_{i}\right)-\log \left(A_{0}\right)$, $\mathrm{X}$ is the $6 \times 1$ vector of unknowns $\left(b D_{i j}\right), G$ is the $N \times 6$ matrix resulting from the concatenation of each row in eq. (11), $\left[g_{i, 1}^{2}, \ldots, g_{i, 3}^{2}\right]$, and $W$ is the $N \times N$ matrix of weights. The Gauss-Markov theorem states that under very weak assumptions the WLS is the minimum variance estimate if $W$ is chosen to be the inverse of the covariance matrix of data, $C_{\mathbf{Y Y}}$. If the estimator is unbiased (as is approximately the case for Rician noise), WLS is in fact the BLUE. Since the noise in each gradient image is assumed to be independent, $C_{\mathbf{Y Y}}$ reduces to a diagonal matrix with diagonal elements equal to the variance of each term $\log \left(M_{i}\right)-\log \left(A_{0}\right)$, which is the same as the variance of $\log \left(M_{i}\right)$ since we consider $A_{0}$ known. Therefore, $W=\operatorname{diag}\left(W_{i i}\right)$ with:

$$
W_{i i}=\operatorname{Var}^{-1}\left\{\mathbf{Y}_{i}\right\} \simeq \frac{1}{a_{i}^{-1} / 2-a_{i}^{-2}(3 L-4) / 4} \simeq 2 a_{i}+(3 L-4)
$$

However, we are going to neglect the terms of order $a_{i}^{-2}$, so that we will fix $W_{i i}=2 a_{i}=A_{L_{i}}^{2} / \sigma^{2}$. We do so for two reasons:

1. This formulation is identical to the traditional WLS for Rician noise, see 1, and is the one implemented in all the software tools for DT estimation. Note that even in the case of Rician noise the term in $a^{-2}$ is not null (but very small), and even so the DT is always estimated with this formulation.

2 . The weights $W_{i i}$ in fact have to be only proportional to $2 a_{i}$, so with this formulation it is not necessary to know the exact value of $\sigma$, since we may take any weight proportional to $A_{L_{i}}^{2}$; if we include the term in $a^{-2}$, it is necessary to estimate $\sigma$ at each image location and to include the number of coils, $L$, as an additional parameter of the algorithm.

Once again, the whole analysis may be performed with the exact expression for $W_{i i}$ with very similar conclusions. Finally, note that the weights $W_{i i}$ depend on $A_{L i}$ (not $M_{i}$ ), so they cannot be known a priori; this pitfall may be obviated by iteratively estimating $\mathbf{X}$ and updating the value of $W[1$. Therefore, in what follows we will consider that $W_{i i}$ are known without uncertainty.

\section{Variance and Bias of the Tensor Components}

In what follows, we are going to call $\mathcal{L}=\left(G^{T} W G\right)^{-1}$. Then, from eqs. (9), (10) and (12), and with our election of $W_{i i}$ the covariance matrix of $\mathbf{X}$ is:

$$
C_{\mathbf{X X}}=E\left\{(\mathbf{X}-E\{\mathbf{X}\})(\mathbf{X}-E\{\mathbf{X}\})^{T}\right\}=\mathcal{L} G^{T} W C_{\mathbf{Y} \mathbf{Y}} W^{T} G \mathcal{L}^{T}
$$




$$
\begin{aligned}
& =\mathcal{L} G^{T} W \operatorname{diag}\left(\frac{1}{2} a_{i}^{-1}-\frac{\Theta(L)}{4} a_{i}^{-2}\right) W G \mathcal{L} \\
& =\mathcal{L} G^{T} W G \mathcal{L}-\frac{\Theta(L)}{4} \mathcal{L} G^{T} W \operatorname{diag}\left(a_{i}^{-2}\right) W G \mathcal{L}=\mathcal{L}-\Theta(L) \mathcal{L} G^{T} G \mathcal{L}
\end{aligned}
$$

where $\Theta(L)=3 L-4$ and $W, \mathcal{L}$ are symmetric. Note that the second term in eq. (14) appears due to the fact that we are using $W_{i i}=2 a_{i}$ instead of the true value of $\operatorname{Var}^{-1}\left\{Y_{i}\right\}$, and it is not null even for the Rician case (yet, $\Theta(L)$ is negative for $L=1$, so for the Rician case the variance is greater than $\mathcal{L}$, contrary to the case of $L>1)$. For the bias, since $\operatorname{bias}\left\{\mathbf{Y}_{i}\right\}=a_{i}^{-1}(L-1) / 2$, we have:

$$
\operatorname{bias}\{\mathbf{X}\}=\left(G^{T} W G\right)^{-1} G^{T} W \operatorname{bias}\{\mathbf{Y}\}=(L-1) \mathcal{L} G^{T} \mathbf{e}_{1}
$$

where $\mathbf{e}_{1}$ is an $N \times 1$ vector of ones. For the $6 \times 1$ vector $G^{T} \mathbf{e}_{1}$, let us assume that the gradient directions are uniformly distributed on the sphere; each component $g_{k}, k=1,2,3$ may be seen as a uniform random variable, $g_{k} \sim U(-1,1)$, so that $\sum_{i=1}^{N} g_{i, k}^{2} \equiv N E\left\{g_{k}^{2}\right\}=N / 3, \sum_{i=1}^{N} 2 g_{i, j} g_{i, k} \equiv 2 N E\left\{g_{j}\right\} E\left\{g_{k}\right\}=0$ and $G^{T} \mathbf{e}_{1}=[N / 3,0,0, N / 3,0, N / 3]^{T}=N \nu$. Although this reasoning may seem quite naive, we have checked this result to be extremely accurate for all $N$ in the case of antipodal symmetric gradients. Finally, we define the MSE as:

$$
\begin{aligned}
\mathrm{MSE} & =\operatorname{Var}\{\mathbf{X}\}+\operatorname{bias}^{2}\{\mathbf{X}\}=b^{2}\left(\Delta_{11}^{2}+\Delta_{12}^{2}+\Delta_{13}^{2}+\Delta_{22}^{2}+\Delta_{23}^{2}+\Delta_{33}^{2}\right) \\
& =\operatorname{tr}(\mathcal{L})-\Theta(L) \operatorname{tr}\left(\mathcal{L} G^{T} G \mathcal{L}\right)+(L-1)^{2} N^{2} \nu^{T} \mathcal{L}^{2} \nu
\end{aligned}
$$

for $\Delta_{i j}=D_{i j}-\widetilde{D}_{i j}$ and $\widetilde{D}_{i j}$ the WLS estimate of $D_{i j}$.

\section{Results and Discussion}

To begin with, we will verify the accuracy of the approximations given by eqs. (9) and (10): in Fig. 1 (left) we represent the true values given by eqs. (4) and (5), together with empirically obtained values (for 2000 independent samples for each $a$ and $L$ ), superimposed to the proposed approximations. As may be seen, these approximations are very accurate except for very low (unrealistic) values of $a$; the larger $L$, the larger the value of $a$ below which the approximation diverges from the actual value. Nevertheless, note that the difference is noticeable only for $L=8$ (below $a=60$ ). As shown later on (and in Fig. 1, right) for $L=8$ the values of interest for $a$ are in the order of 140; for larger values of $L$, the approximations are inexact for higher $a$, but at the same time the study of the bias is of interest for higher $a$, so our approximation is good enough in all cases.

We now assess the impact of the bias compared to the variance in the components of the DT. First, let us assume a simplified scenario where all $W_{i i}$ are equal to $2 a_{i}=2 a$; then the MSE reduces to:

$$
\operatorname{MSE}=\left(\frac{a^{-1}}{2}-\frac{a^{-2}}{4} \Theta(L)\right) \operatorname{tr}\left(\left(G^{T} G\right)^{-1}\right)+a^{-2} \frac{(L-1)^{2} N^{2}}{4} \nu^{T}\left(G^{T} G\right)^{-2} \nu
$$



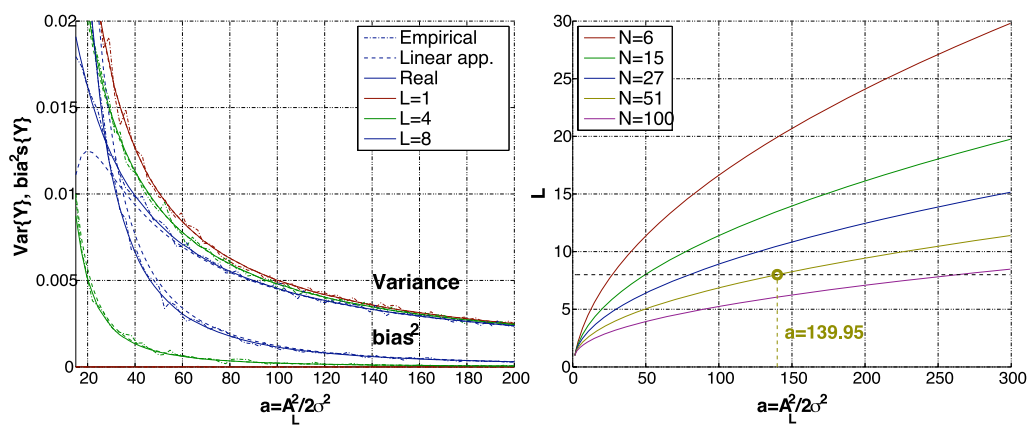

Fig. 1. Left: bias and variance in the DWI signals as a function of $a$ for different numbers of coils; we represent true and empirically computed values together with our approximations. Right: minimum number of receiving coils required (for each $a$ and $N$ ) so that the (squared) bias equals the variance in the DT components (see eq. (18)).

since $\mathcal{L}=a^{-1} / 2\left(G^{T} G\right)^{-1}$. Therefore it is interesting to study the behavior of $G^{T} G$; a similar reasoning to that made for the calculation of $\nu$ shows that it always has the eigenvector $\nu /\|\nu\|$ associated to the eigenvalue $N / 3$ for antipodal symmetric gradient directions. On the other hand, we have empirically tested that the approximation: $\operatorname{tr}\left(\left(G^{T} G\right)^{-1}\right) \simeq 29.3 / N$ is very accurate for all values of $N$. Therefore, we may write:

$$
\begin{aligned}
\mathrm{MSE} & \simeq \frac{29.3}{N}\left(\frac{a^{-1}}{2}-\frac{a^{-2}}{4}(3 L-4)\right)+a^{-2} \frac{9(L-1)^{2} N^{2}}{4 N^{2}} \nu^{T} \frac{\nu \nu^{T}}{\|\nu\|^{2}} \nu \\
& =\frac{29.3}{N}\left(\frac{a^{-1}}{2}-\frac{a^{-2}}{4}(3 L-4)\right)+a^{-2} \frac{3(L-1)^{2}}{4}
\end{aligned}
$$

since $\left(G^{T} G\right)^{-2}$ has the eigenvector $\nu /\|\nu\|$ associated to $9 / N^{2}$ and all other eigenvectors are orthogonal to $\nu$. While the variance diminishes with the number of gradients, this is not the case for the bias: in Fig. 1 (right) we show the number of coils needed for each $a$ and $N$ so that the term corresponding to the bias in eq. (18) equals the term corresponding to the variance. For example, with $L=8$ coils and 51 gradient directions, it is shown that if $a<139.95$ the bias is more important than the variance. Alternatively, for $a<139.95$ and $L=8$ it makes little sense to take more than 51 gradients, since the variance is reduced but not the bias, so the acquisition time is increased unfruitfully; for $a=50, L=8$, the situation is the same for more than 15 gradients. Therefore, it may be more convenient to increase the number of repetitions (NEX) in the acquisition; this has the effect of dividing the noise power $\sigma^{2}$ by NEX (since the signals are averaged in the $\mathbf{k}$-space, where the noise is Gaussian distributed), so that:

$$
\mathrm{MSE}=\frac{29.3}{N \cdot \mathrm{NEX}}\left(\frac{a^{-1}}{2}-\frac{a^{-2}}{4 \mathrm{NEX}}(3 L-4)\right)+a^{-2} \frac{3(L-1)^{2}}{4 \mathrm{NEX}^{2}}
$$

A constant value of $N$. NEX takes a constant amount of acquisition time, and besides provides a constant reduction factor for the variance, but the bias 

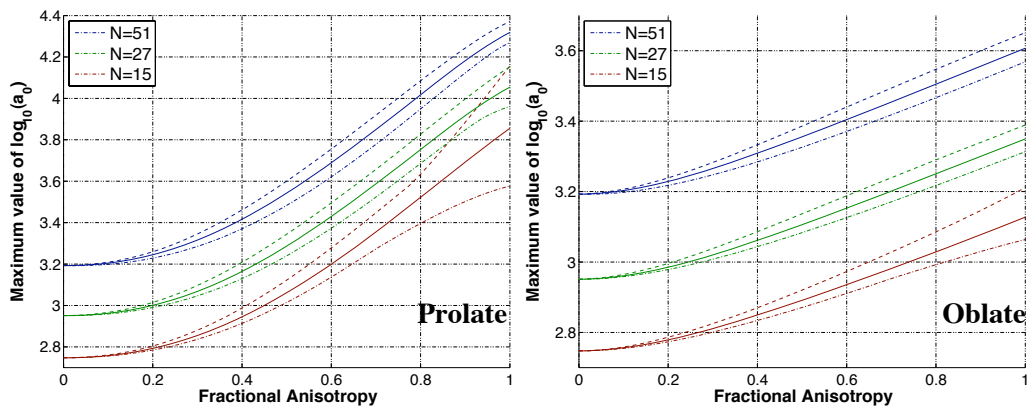

Fig. 2. $\log$-plot of the maximum value of $a_{0}=L A_{0}^{2} / 2 \sigma^{2}$ which makes the squared bias equal to the variance in the components of the DT for different tensor shapes. We show minimum, mean and maximum values among all possible tensor orientations.

depends only on NEX. We may conclude that, for a given combination of $a, N$ and $L$ which makes the squared bias similar to the variance (see Fig. 1), it is preferable to increase NEX than using more gradient directions.

As a final experiment, we test the behavior when the weigths $W_{i i}$ are not equal (non-isotropic diffusion). To do so, we use typical values $L=8, N=$ 15,27 , and $51, b=1500 \mathrm{~s} / \mathrm{mm}^{2}$, and Mean Diffusivity (MD) $0.8 \cdot 10^{-3} \mathrm{~mm}^{2} / \mathrm{s}$, and compute the maximum value of $a_{0}$ (defined as $a_{0}=A_{L_{0}}^{2} / 2 \sigma^{2}$ with $A_{L 0}$ the amplitude of the baseline image) for which the contribution of the bias is greater or equal than the contribution of the variance, using eq. (16). Since this result depends on the orientation of the DT, in Fig. 2 we show maximum, minimum and mean values of $a_{0}$ for different Fractional Anisotropies (FA). Two cases are considered: a prolate tensor $\left(\lambda_{1}>\lambda_{2}=\lambda_{3}\right)$ and an oblate tensor $\left(\lambda_{1}=\lambda_{2}>\lambda_{3}\right)$. For $\mathrm{FA}=0$ (isotropic diffusion, equal weights), we have the same case as in Fig. 1 (right). With non-isotropic tensors the bias becomes even more important $\left(a_{0}\right.$ may be one order of magnitude over the value for $\mathrm{FA}=0$ with the prolate tensor), so the conclusions arised from our previous experiment are even reinforced. For example, with 51 directions and a prolate tensor with $\mathrm{FA}=0.8$, even for $a_{0}=10^{4}\left(A_{L_{0}} / \sigma \simeq 140\right)$ the bias is as important as the variance; although this corresponds to the baseline image, which typically shows a high SNR, it is evident that this value may be found in many image voxels. Besides, for $A_{L_{0}} / \sigma \simeq 300$ (this SNR is an upper limit for a realistic DWI data set) the bias will be roughly $1 / 4$ of the variance, which is clearly not negligible.

\section{Conclusions}

We have shown that the impact of the bias in Rician signals for WLS tensorfitting may be neglected in any realistic case; on the contrary, for non-central $\chi$ distributed signals, the bias may be an important source of error, the larger the number of receiving coils $L$ the more critical. While the variance in the estimation may be reduced increasing the number of gradient directions, this is not 
the case for the bias. In some cases, increasing the number of gradients will not improve the estimation, since the main source of error will be the bias and not the variance. In these cases, it may be preferable to improve the SNR by increasing NEX (unless a minimum number of gradient directions is needed for some purpose). As an additional difficulty, we have shown that the traditional WLS approach is not optimal for non-central $\chi$ signals, since the weights commonly used are not those yielding minimum variance; although we have proposed a modification to avoid this problem, it makes necessary to characterize the noise power for all image voxels. Summarizing, the importance of the bias in noncentral $\chi$ distributed DWI has been stated; this kind of statistics are usually found in multiple receiving coils systems with SoS reconstruction, but may be used as well to model some pMRI algortihms like GRAPPA. Therefore, an adequate unbiased filtering scheme (or a high NEX, increasing the acquisition time) should be implemented before DT estimation with modern DT-MRI protocols.

Acknowledgments. Work partially funded by grant numbers TEC2007-67073/ TCM from the CICyT (Spain) and NIH R01 MH074794, NIH P41 RR13218.

\section{References}

1. Salvador, R., Pea, A., Menon, D.K., Carpenter, T.A., Pickard, J.D., Bullmore, E.T.: Formal characterization and extension of the linearized diffusion tensor model. Human Brain Mapping 24, 144-155 (2005)

2. Fillard, P., Pennec, X., Arsigny, V., Ayache, N.: Clinical DT-MRI estimation, smoothing, and fiber tracking with $\log$-euclidean metrics. IEEE Trans. Med. Imaging 26(11), 1472-1482 (2007)

3. Gudbjartsson, H., Patz, S.: The Rician distribution of noisy MRI data. Magn. Reson. Med. 34, 910-914 (1995)

4. Roemer, P., Edelstein, W., Hayes, C., Souza, S., Mueller, O.: The NMR phased array. Magn. Reson. Med. 16, 192-225 (1990)

5. Constantinides, C., Atalar, E., McVeigh, E.: Signal-to-noise measurements in magnitude images from NMR phased arrays. Magn. Reson. Med. 38, 852-857 (1997)

6. Gilbert, G., Simard, D., Beaudoin, G.: Impact of an improved combination of signal from array coils in diffusion tensor imaging. IEEE Trans. Med. Imaging 26(11), 1428-1436 (2007)

7. Aja-Fernández, S., Alberola-López, C., Westin, C.-F.: Signal LMMSE estimation from multiple samples in MRI and DT-MRI. In: Ayache, N., Ourselin, S., Maeder, A. (eds.) MICCAI 2007, Part II. LNCS, vol. 4792, pp. 368-375. Springer, Heidelberg (2007)

8. Basu, S., Fletcher, T., Whitaker, R.T.: Rician noise removal in diffusion tensor MRI. In: Larsen, R., Nielsen, M., Sporring, J. (eds.) MICCAI 2006. LNCS, vol. 4190, pp. 117-125. Springer, Heidelberg (2006)

9. Descoteaux, M., Wiest-Daesslé, N., Prima, S., Barillot, C., Deriche, R.: Impact of rician adapted non-local means filtering on HARDI. In: Metaxas, D., Axel, L., Fichtinger, G., Székely, G. (eds.) MICCAI 2008, Part II. LNCS, vol. 5242, pp. 122-130. Springer, Heidelberg (2008)

10. Wiest-Daesslé, N., Prima, S., Coupé, P., Morrissey, S.P., Barillot, C.: Rician noise removal by non-local means filtering for low signal-to-noise ratio MRI: Applications to DT-MRI. In: Metaxas, D., Axel, L., Fichtinger, G., Székely, G. (eds.) MICCAI 2008, Part II. LNCS, vol. 5242, pp. 171-179. Springer, Heidelberg (2008)

11. Stejskal, E.O., Tanner, J.E.: Spin diffusion measurements: Spin echoes in the presence of a time-dependent field gradient. J. of Chem. Phys. 42, 288-292 (1965) 